\section{TREATMENT OF DEEP BITE MALOCCLUSIONS}

Align Technology, Inc. has announced the release of Invisalign ${ }^{\text {G5 }}$ Innovations for Deep Bite in the UK. These new innovations are specifically designed for the treatment of deep bite malocclusions and have been engineered to help practitioners achieve even better clinical outcomes when treating deep bite.

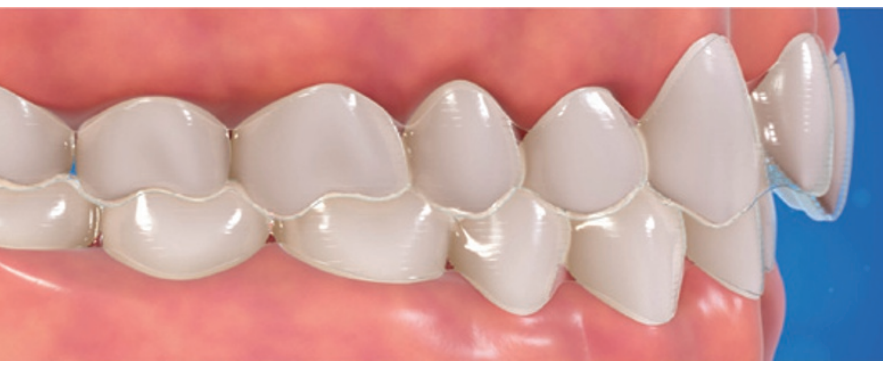

The new features to help achieve this include:

- New SmartForce attachments and Pressure Areas designed to level the curve of Spee by improving control of premolar extrusion and anterior intrusion for more predictable deep bite treatments

- Precision Bite Ramps designed to disocclude the posterior teeth for improved efficiency in deep bite treatments.

These features are engineered to deliver the forces needed to achieve predictable tooth movements with

\section{FIFTY YEARS OF EQUIPMENT MANUFACTURING}

Founded in 1964, A-dec celebrates a half-century of 'quality through caring' with gratitude for customers and dealers, suppliers, and the community at-large.

Today, A-dec's 50-acre Newberg headquarters is home to more than 1,000 employees who manufacture and market equipment to dental professionals worldwide. A-dec also has facilities in the UK, Australia, and China, which support an extensive network of authorised dealers in more than 100 countries worldwide.

A-dec views the anniversary as an opportunity for employees to inspire one another, and to ensure its reputation for quality continues to flourish.

The company pioneered many of the innovations used in dental treatment rooms today, which include the evolution of patient chairs, delivery systems, and dental lights.

Co-founder Ken and the late Joan Austin were responsible for producing and patenting dentistry's first vacuum saliva ejector, which served an important role in the advent of sit-down dentistry. The 1964 innovation would replace the era's cumbersome belt-drive devices.

A-dec also introduced the first compact delivery system, revolutionising how dentists are able to treat patients more efficiently and ergonomically.

Ken Austin continues to be involved with portable equipment designed for mobilising treatment to under-served populations.

www.a-dec.co.uk

\section{FOR GUMS TO BE PROUD OF}

Working closely with the natural oral $\mathrm{pH}$, the UltraDEX Performance Oral Care range may be of value to a wide spectrum of oral hygiene concerns when used as part of an oral hygiene regime. The clinically proven $\mathrm{iQ}+$ ActiveOxi Technology with activated stabilised chlorine dioxide helps to prevent plaque biofilm, inhibits its re-growth and instantly eliminates bad breath compounds for at least 12 hours. There are no unpleasant side effects such
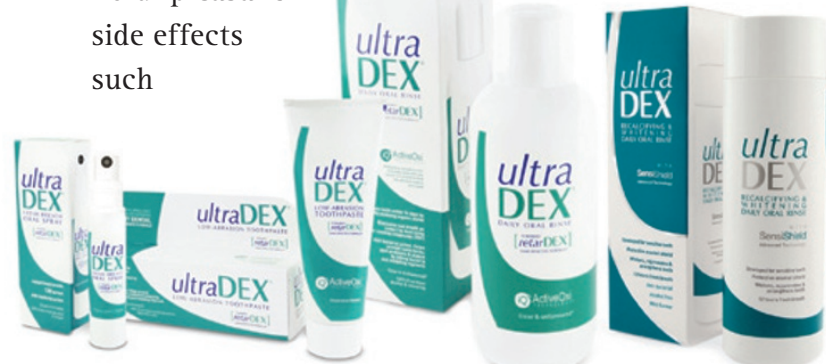

as staining or interference with taste sensation.

This technology also gently lifts off organic stains such as tea, coffee and red wine by a process of oxidation, leaving teeth whiter and brighter. Without alcohol and free from sodium lauryl sulphate, the range is gentle and safe for long-term daily use, bridging the gap between professional and at-home care. For further ultra
DEX ultraDEX $\quad$ www.periproducts. ultraDEX www.per
co.uk or email ultraDEX 1=oxi dental@periproducts.co.uk.
Invisalign. Based on biomechanical principles, SmartForce features are customised for each tooth using advanced virtual modelling and are positioned precisely to deliver the proper forces.

Invisalign ${ }^{65}$ Innovations for Deep Bite are available on all Invisalign treatment options at no additional cost to Invisalign-trained providers worldwide from 24 February 2014 and showcased at a masterclass for advanced providers on 22 March in London with Drs Sam Daher and Dai Roberts-Harry.

www.invisalign.com

\section{BROWSE IMAGING PRODUCTS ON THE GO}

MyRay is renowned for producing some of the highest quality imaging systems and dental radiology equipment in the industry. Offering the perfect combination of simplicity and performance, all technical solutions are designed specifically for the modern profession.

The MyRay Dental Imaging app is now also available to provide you with all the information you could need, for free.

Find product descriptions, videos, troubleshooting protocols and order forms all in the one app, ensuring you purchase the right equipment for you.

Available for iPad and iPhone, the app enables you to browse MyRay products at a time and place that is convenient to you, whether that is at work, at home or on the train.

With new apps and more information coming soon, make sure you don't miss out and download the free MyRay Dental Imaging app today.

Tavom UK is the distributor of MyRay Products in the UK. To find out more visit www.tavomuk.com or call 0870 7521121. 